Original article

\title{
Metacognitive Training for Depression (D-MCT) reduces false memories in depression. A randomized controlled trial
}

\author{
Steffen Moritz ${ }^{1, *}$, Brooke C. Schneider ${ }^{1}$, Judith Peth, Sönke Arlt, Lena Jelinek \\ Department of Psychiatry and Psychotherapy, University Medical Center Hamburg-Eppendorf, Martinistraße 52, 20246 Hamburg, Germany
}

\section{A R T I C L E I N F O}

\section{Article history:}

Received 4 April 2018

Received in revised form 8 May 2018

Accepted 24 May 2018

Available online 8 June 2018

\section{Keywords:}

Depression

False memory

Memory

Deese-Roediger-McDermott paradigm

Metacognitive training

\section{A B S T R A C T}

Metacognitive Training for Depression (D-MCT) is a highly standardized group program targeted at depression-related ("Beckian") emotional as well as cognitive biases, including mood-congruent and false memory. While prior results are promising with respect to psychopathological outcomes (depression), it is unclear whether D-MCT also meets its goal of improving cognitive biases, such as false memories.

In the framework of a randomized controlled trial (registered trial, DRKS00007907), we investigated whether D-MCT is superior to an active control condition (health training, HT) in reducing the susceptibility of depressed patients for false memories. False memories were examined using parallel versions of a visual variant of the Deese-Roediger McDermott paradigm.

Both groups committed less false memories at post assessment after 4 weeks compared to baseline. Relative to HT, D-MCT led to a significant decrease in high-confident false memories over time.

The study presents first evidence that D-MCT decreases the susceptibility of depressed patients for false memories, particularly for errors made with high confidence that are presumably the most "toxic" in terms of mood-congruent memory distortions.

(c) 2018 Elsevier Masson SAS. All rights reserved.

\section{Introduction}

Major depression is one of the most prevalent and debilitating psychiatric disorders [1] and ranks 5th worldwide among the leading causes of years lived with disability [2]. It affects one in seven persons at some point during their life, with a lifetime morbidity risk of approximately 30\% [3]. The disorder is multidimensional and involves cognitive (e.g., concentration difficulties), somatic (e.g., disrupted sleep), and emotional symptoms (e.g., feelings of guilt and/or worthlessness) and most individuals with a depressive disorder experience some difficulties in social, occupational and home functioning [4,5].

Whereas memory dysfunctions are not among the core diagnostic features of depression, they are prevalent [6] and represent a multi-faceted and important battleground for the disorder. In addition to "cold" memory deficits for retrospective $[7,6]$ and prospective material $[8]$ that manifest already at the first episode [9], a number of memory distortions have been elucidated in depression that likely play a role in both the formation and

\footnotetext{
* Corresponding author.

E-mail address: moritz@uke.de (S. Moritz).

1 Split first author.
}

maintenance of the disorder. For quite some time now, overgeneralized autobiographical memory (OGM) has been implicated as an important cognitive contributor to depression. OGM is associated with depression severity [10] as well as an increased risk for disease chronicity [11-15]. Another well-researched cognitive bias that has attracted attention since the 1980 s $[16,17]$ is the moodcongruent memory (MCM) effect [18], which refers to the phenomenon that depressed patients often show better recall and recognition for negative-valenced memory material $[19,20]$. It has been argued that this bias is most prominent for negative information that is personally relevant $[18,19,21]$.

In addition, the phenomenon of false memories has been investigated in depression. False memories are defined as the recall of objects that were never presented, or events that never occurred [22]. Several studies have demonstrated that depression is not only characterized by enhanced loss of memory information, but also an increased susceptibility for false memories [20,21,24-26]. Importantly, false memories, as retrospective memories, are often moodcongruent: Patients or individuals with a negatively-induced mood are more prone to falsely remember negative information $[21,23,25-28]$. More recently, it was found that MCM is modulated by context, as it makes a difference whether a positive word like "love" is presented with a positive or negative adjective (e.g., "no" or "much") [25,26,29]. 
The aforementioned memory distortions may lower mood in multiple ways. "Cold" (or content-neutral) cognitive dysfunction is related to psychosocial and functional outcome [30,31]) and cognitive dysfunction may be a primary mediator of functional deficits observed in depression [5,32]. For example, low work performance is likely to lower mood via enhanced stress and other negative consequences (e.g., working over-time). However, some cognitive dysfunctions are secondary (see below) and subjective perception of decreased memory performance is more pronounced than objective problems [33]. The former may foster unfounded worry about developing dementia or neurodegeneration, especially in middle-aged or older patients, which can foster depressive symptoms by catastrophizing [34]. Memory preference for negative material may thus lead to a preponderance of negative information in an individuals' consciousness and heavily distorts one's view of him- or herself and others, as well as the future [i.e., cognitive triad of depression; 35]. Accordingly, improving memory performance and "correcting" memory biases may also represent an important mechanism for mood repair. We thus concur with recent calls $[35,36]$ to make cognitive problems a core target of depression treatment.

Psychotherapy, and particularly cognitive behavioral therapy (CBT), has shown to be effective in the treatment of depression, including relapse prevention [37,38]. CBT targets cognitiveaffective biases, such as selective abstraction and over-generalization. In contrast, teaching patients about the aforementioned memory problems and biases is not part of standard CBT programs. Metacognitive Training for Depression (D-MCT) aims to fill this gap [39]. D-MCT amalgamates CBT exercises with a metacognitive approach that aims to modify depression-related "Beckian" emotional, as well as more general cognitive biases. D-MCT is a highly standardized open group intervention that contains eight modules. It is rooted in MCT for psychosis; for the similarities and differences between D-MCT and metacognitive therapy by Wells' please see a recent commentary [40]. One of D-MCT's eight modules is devoted to memory. Patients are educated about memory problems in depression (and their origin), are introduced to the phenomenon of mood-congruent (false) memories and are taught how to improve their memories. It also addresses the frequent worry of patients that they may develop dementia. As discussed above, while memory impairment and other neuropsychological dysfunctions frequently occur in depression, the module uses a psychoeducational approach to inform patients that such memory difficulties may also be secondary to reduced motivation, or anxiety, as well as rumination or concentration difficulties during formal assessments [41,42]. Disclosure of these secondary influences is intended to attenuate patients' exaggerated worries about a neurodegenerative process (normalization).
Results from a pilot study revealed positive effects of D-MCT on depressive symptoms [43]. Similar results were reported in a randomized controlled trial (RCT), in which D-MCT was found to be superior compared to an active control group with medium to strong effect sizes [44]. Additionally, patients rate the training positively at both immediate and long-term assessments [45].

This is the first study to investigate the influence of D-MCT on the proneness for false memories in depressed patients. We hypothesize that in comparison to a control intervention (i.e., Health Training), D-MCT would yield a greater effect in reducing patients' susceptibility for false memories from baseline to postassessment after 4 weeks.

\section{Methods}

\subsection{Participants}

This study is part of a larger trial [44], which reports results on the primary outcomes [i.e., measures of depression, dysfunctional attitudes, self-esteem and overall functioning] but not on false memories. A total of 84 patients who fulfilled DSM-IV diagnostic criteria for a depressive disorder and had no history of psychotic symptoms (i.e., hallucinations, delusions, or mania), current suicidality (Suicidal Behaviors Questionnaire Revised score $\geq 7$ ), or intellectual disability (estimated IQ $<70$ ). Patients were recruited from a psychosomatic outpatient day clinic (RehaCentrum Hamburg) at the University Medical Center HamburgEppendorf (Germany). Patients were randomly allocated to either Metacognitive Training for Depression (D-MCT; $n=41$ ) or Health Training (HT; $n=43$ ). Written informed consent was obtained from all participants before participation in the baseline assessment. The study was approved by the Ethics Committee of the German Association for Psychology (DGPs) and was conducted in accordance with the Declaration of Helsinki. For the current study, participants with substance or alcohol dependence, as well as patients with a neurological disorder (including brain injury) were excluded. A total of 77 participants underwent the false memory paradigm at pre-assessment (92\% from entire sample) and 68 at post-assessment [88\% retention rate from subsample; D-MCT, $n=39$ (pre), $n=33$ (post); health training, $n=38$ (pre), $n=35$ (post)]. Table 1 shows group comparisons at baseline. Individuals in the two condition did not differ on any psychopathological or background characteristics, including type of medication, number of episodes and comorbid diagnoses. All participants provided written informed consent prior to participation. The study was registered at the German Clinical Trials Register (No. DRKS00007907).

Table 1

Sociodemographic and Psychopathological Characteristics of the Sample. Frequencies, Means and Standard Deviations (in Brackets).

\begin{tabular}{|c|c|c|c|}
\hline Variable & D-MCT $(n=39)$ & $\mathrm{HT}(\mathrm{n}=38)$ & Statistics \\
\hline \multicolumn{4}{|l|}{ Background characteristics } \\
\hline Age & $44.28(10.11)$ & $47.68(9.12)$ & $t(75)=1.55 ; p=.126$ \\
\hline Gender (female/male) & $28 / 11$ & $29 / 9$ & $\chi^{2}(1)=0.20, p=.651$ \\
\hline Education (years) & $10.51(1.64)$ & $10.43(1.54)$ & $t(75)=0.22 ; p=.829$ \\
\hline IQ (MWT-B) & $107.24(12.12)$ & $109.97(12.13)$ & $t(72)=0.97 ; p=.335$ \\
\hline \multicolumn{4}{|l|}{ Treatment and Diagnoses } \\
\hline Medication (antidepressant, antipsychotic, combination, none) & $26 / 0 / 1 / 12$ & $24 / 2 / 1 / 11$ & $\chi^{2}(3)=2.11, p=.550$ \\
\hline Presence of dysthymia & $28 / 10$ & $28 / 10$ & $\chi^{2}(1)=0.00, p>.99$ \\
\hline Presence of comorbid anxiety disorder & $20 / 19$ & $21 / 17$ & $\chi^{2}(1)=0.12, p=.726$ \\
\hline Numbers of depressive episodes & $4.21(8.01)$ & $2.00(2.18)$ & $t(64)=1.53 ; p=.134$ \\
\hline \multicolumn{4}{|l|}{ Psychopathology } \\
\hline BDI-I & $25.28(8.57)$ & $27.22(11.85)$ & $t(74)=0.81 ; p=.420$ \\
\hline HDRS & $16.59(5.01)$ & $17.84(6.78)$ & $t(75)=0.92 ; p=.361$ \\
\hline
\end{tabular}

Notes: BDI-I = Beck Depression Inventory-I, HDRS = Hamilton Depression Rating Scale, MWT-B = vocabulary test to estimate verbal intelligence. 


\subsection{Measures}

\subsubsection{Psychopathology}

At baseline, each patient was interviewed with the Mini International Neuropsychiatric Interview (M.I.N.I) [46] to verify the presence of a single episode of major depression, recurrent depression, or dysthymia according to DSM-IV criteria. The 17item version of the Hamilton Depression Rating Scale (HDRS) [47] was used to quantify depression at both assessment points. While the HDRS represents the gold standard for most clinical trials on depression, it has faced a number of criticisms [48,49] so that depression was also captured with a self-rating instrument, the Beck Depression Inventory-I (BDI-I) [50,51].

\subsubsection{False memory paradigm}

The false memory effect was initially demonstrated for word lists [52,22], but subsequent research has replicated the phenomenon for visual [53] and other modalities [54]. False memories were assessed with an established visual DeeseRoediger McDermott (DRM) paradigm [55-58] for pre- and postassessment; for details on the development of the task please refer to the respective literature [55-58]. Each picture showed a complex scenario (i.e., "classroom", "funeral", "surveillance", "beach", "Christmas" "construction site"), in which eight details typical/central for the scenario were missing (e.g., in the beach scene, waves and a ball were not pictured). The missing, but strongly implied objects, represented "critical lures" for the recognition phase. A fixed choice of two scenarios were presented at each assessment in randomized order (i.e., "classroom" and "funeral", "surveillance" and "beach", "Christmas" and "construction site"). Which pair of scenes was shown at which assessment time was additionally randomized across participants. Each person was seated approximately 20 inches away from a 15inch computer monitor and was instructed to carefully attend to the picture on the screen. Each of the two pictures was presented individually for $40 \mathrm{~s}$ and filled the entire screen. After the encoding phase, participants were asked to complete a vocabulary test to estimate verbal intelligence as a filler task [59], which lasted approximately $10 \mathrm{~min}$. During the recognition phase, participants were presented studied, "old" items (e.g., people with sunglasses in the beach scene) and two types of non-studied items, presented as single words on the screen. Non-studied were either unrelated "new" items (e.g., "television" for the beach scene) or critical lures (e.g., "beach towel" for the beach scene). The specific order of items was randomized; a contextual cue was provided on the screen (i.e., "classroom", "beach", "funeral", "surveillance", "Christmas", "construction site") to clarify to which scene an item referred to. For each scenario, 24 items were presented: 12 "old" items (previously presented in the picture), 4 unrelated "new" items (not present in the picture and unrelated to the scene), and 8 "critical lures" (items not presented in the picture, but strongly related to the scene). Participants had to rate whether an item was old or new along with memory confidence on a four-point scale: 1 = old, confident, $2=$ old, not confident, 3 = new, confident, 4 = new, not confident.

\subsection{Design}

Patients were randomly allocated to either D-MCT or HT following a fixed randomization plan. Groups met twice a week and the post-assessment occurred 4 weeks after baseline. The DMCT intervention strictly followed the D-MCT manual [39]. The HT intervention was also performed twice weekly in a group format. Each week, patients participated in a psychoeducational group session, which provided information about general health topics (e.g., physical activity, nutrition, sleep) and included a session with physical activity (i.e., walking). At post-assessment, participants again completed all measures administered at baseline (except for the M.I.N.I.), including the visual DRM paradigm. The chosen scenarios for the DRM task were randomized over assessments (pre, post) to reduce practice effects.

\subsection{Statistical analyses}

Baseline differences between treatment groups regarding socio-demographic and psychopathological symptoms were examined by $t$-tests for independent samples. BDI data at baseline was missing for one patient. Patients with missing data on the DRM were excluded. To investigate changes in false memory performance over time, two different analyses were performed. First, a $3 \times 2 \times 2$ ANOVA was carried out with the within-subject factors DRM Item Type ("old”, “new”, “critical lures") and Time (pre, post), and the between-subject factor Group (D-MCT, HT) using the "old" ratings (percentage). Second, to investigate the influence of confidence, a $3 \times 2 \times 2 \times 2$ ANOVA with the within-subject factors Item Type ("old", "new", “critical lures"), Response (old, new), and Time (pre, post) as well as the between-subject factor Group (DMCT, HT) was calculated using high-confident ratings only (please note that the measurement of old-confident and new-confident responses is non-redundant). An alpha level of 0.05 (two-tailed) was used for all statistical tests. Effect sizes [60] were estimated with $\eta_{\text {partial }}{ }^{2} ; \quad \eta_{\text {partial }}{ }^{2}=0.01$ designates a small effect, $\eta_{\text {partial }}{ }^{2}=0.06$ designates a medium effect and $\eta_{\text {partial }}{ }^{2}=0.14$ designates a large effect.

\section{Results}

\subsection{Participants}

Participants in the two conditions did not differ on major demographic background variables or psychopathological symptoms (see Table 1). For results on the primary outcomes and remission rates, the reader is referred to the original article [44]. In short, improvement in depressive symptoms (BDI, HDRS) across time were larger in the D-MCT than in the HT group. More patients in the D-MCT group than in the control group remitted according to HDRS criteria (total score $\leq 8$ ), which bordered significance for post-assessment $(p=0.064)$. Psychological well-being (subscale of the WHO QoL BREF) improved to a greater extent in the D-MCT than in the active control condition at post assessment. Emotional ("Beckian") biases, as measured with the Dysfunctional Attitudes Scale (DAS), improved at trend level from pre to post (intention to treat analyses).

\subsection{False memories and memory accuracy}

The mixed $3 \times 2 \times 2$ ANOVA (dependent variable: rating) revealed significant main effects of Item Type, $F(2,66)=628.63$, $p<.001, \eta_{\text {partial }}^{2}=0.905$, and Time, $F(2,66)=977.54, p=.019$, $\eta_{\text {partial }}{ }^{2}=0.081$, which was qualified by an interaction of Item Type $\times$ Time, $F(2,66)=3.42, p=.036, \eta_{\text {partial }}{ }^{2}=.049$. As shown in Fig. 1, most studied items were correctly rated as "old" and most new unrelated items were correctly rated as "new". In contrast and speaking for the validity of the false memory paradigm, critical lures were often misattributed as "old". The significant two-way interaction emerged because the rate of false memories (critical lures) was attenuated over time as confirmed by a paired $t$-test, $t$ (67) =3.57, $\quad p=.001$. The three-way interaction (Item Type $\times$ Group $\times$ Time) revealed a linear trend, $F(2,66)=3.32$, $p=.073, \eta_{\text {partial }}{ }^{2}=0.048$, owing to somewhat less errors made by the D-MCT group across time. 


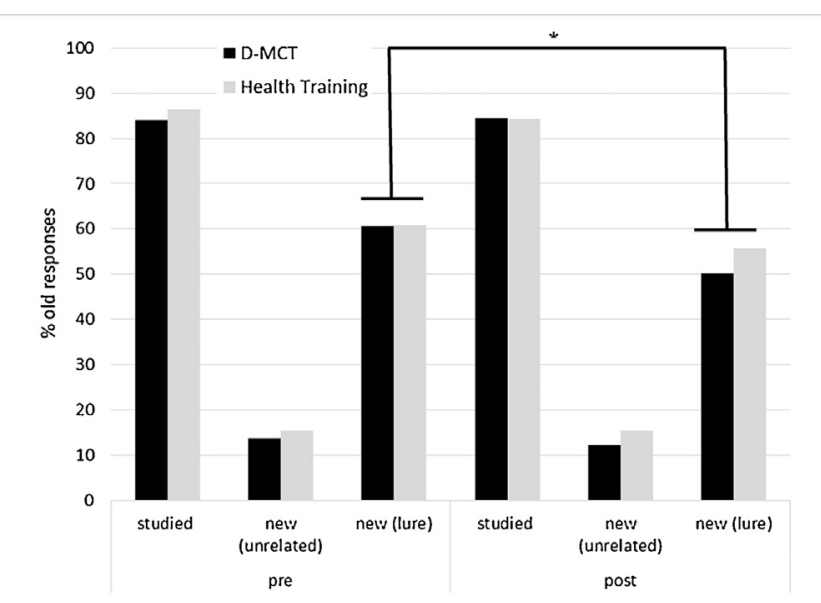

Fig. 1. Ratings classified as "old" in the visual DRM paradigm for studied ("old") items, unrelated new items, as well as "critical lures" across time depicted separately for the experimental (i.e., "Metacognitive Training for Depression" [D-MCT]) and the control intervention (i.e., "Health Training" [HT]). Critical lure items were frequently misclassified as "old" but this tendency decreased significantly over time ( ${ }^{*}$ paired $t$-test: $p<.001$ ).

\subsection{False memories and confidence ratings}

The mixed four-way ANOVA (dependent variable: high-confident judgments) revealed significant main effects for Item Type, $F$ $(2,66)=80.10, p<.001, \eta_{\text {partial }}{ }^{2}=0.548$, and Response (old/new), $F$ $(1,66)=34.72, p<.001, \eta_{\text {partial }}{ }^{2}=0.345$, which were qualified by a significant two-way interaction of Item Type $\times$ Response, $F$ $(2,66)=503.35, p<.001, \eta_{\text {partial }}^{2}=0.884$, as well as the threeway interaction of Item Type $\times$ Response $\times$ Time, $F(2,66)=5.27$, $p=.006, \eta_{\text {partial }}{ }^{2}=0.074$. As in the first ANOVA, old and unrelated new items were mainly attributed correctly (old items: $72 \%$ oldconfident, 6\% new-confident; new-unrelated items: 5\% oldconfident, $49 \%$ new-confident responses), which was not the case for "critical lures" (old-confident: 30\%, new-confident: 18\%). The three-way interaction emerged because for critical lures the rate of high-confident errors (i.e., old) decreased (32\% to $28 \%$ ) while the rate of high-confident correct (i.e., new) increased from $15 \%$ to $22 \%$ over time. For the four-way interaction, which included the Group factor, a significant linear trend emerged, $F(1,66)=5.46, p=.022$, $\eta_{\text {partial }}{ }^{2}=0.076$, which we followed up using single comparisons.

For each item type ("old", "new", "critical lures"), separate $2 \times 2 \times 2$ ANOVAs with the within-subject factors Response (oldconfident, new-confident) and Time (pre, post), as well as the between-subject factor Group (D-MCT, HT) were calculated (see Fig. 1). The mixed $2 \times 2 \times 2$ ANOVA for "critical lures" (Fig. 2A) revealed a significant interaction of Time $\times$ Response $\times$ Group, $F$ $(1,66)=4.63, p=.04, \eta_{\text {partial }}{ }^{2}=.07$. The amount of "old-confident" responses to "critical lures" decreased from pre (D-MCT: 34\%; HT: $30 \%$ ) to post assessment (D-MCT: $25 \%$; HT: $29 \%$ ) to a larger extent in the D-MCT group (the pairwise $t$-test was significant for D-MCT, $t$ $(32)=2.20, p=.035$, but not the active control group, $t(34)=0.69$, $p=.492)$. Likewise, the amount of "new-confident" responses increased from pre (D-MCT: $17 \%$; HT: $12 \%$ ) to post assessment (DMCT: 28\%; HT: 17\%) to a greater extent in the D-MCT relative to the active control condition (this was significant for the D-MCT, $t$ $(32)=4.04, p<.001$, and at a lower magnitude also for the control group, $t(34)=2.11, p=.043$ ). For "old" and unrelated "new" items none of the effects involving Group achieved significance.

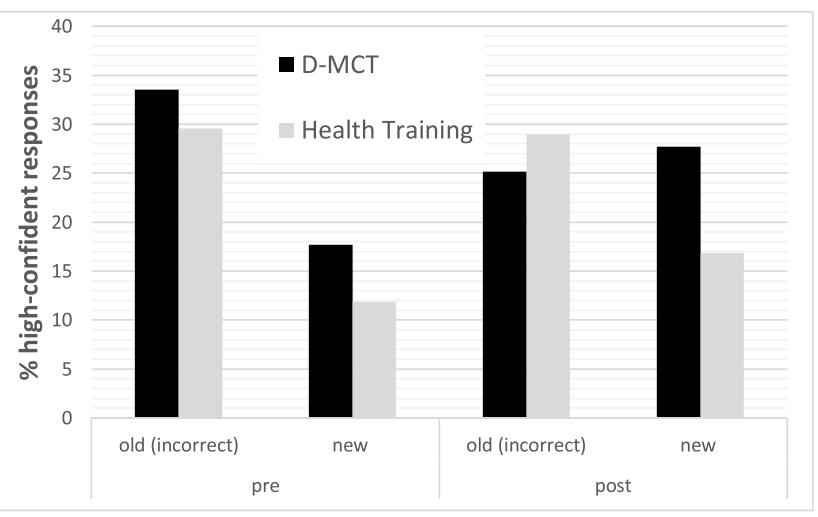

Fig. 2. Percentage of "high-confident responses" in the visual DRM paradigm for critical lure items (correct response: new) during pre and post assessment in depressive patients. In the D-MCT condition, the rate of high-confident false memories decreased more strongly over time relative to the active control condition (D-MCT vs. Health Training).

The decline in false memory (calculated separately for all highconfident false memories and responses made with high confidence) did not correlate with decline on the BDI or the HDRS (all $r s<0.22$, all $p s>.1$ ). Also, none of the memory parameters were correlated with BDI or HDRS scores at baseline $(|r|<0.2 ; p>.1)$. Finally, we examined the possibility that false memories increased during treatment, which was seen for only a small subgroup of patients; $8.6 \%$ of the participants in the control group showed more false memories (irrespective of confidence) at post than at baseline (criterion: increase of more than one false memory over time) and only $6.1 \%$ of the experimental group. The difference was not significant, $\chi^{2}(1)=0.157, p=.692$.

\section{Discussion}

For the first time, we explored whether Metacognitive Training for Depression (D-MCT) reduces the susceptibility of depressed patients for false memories. Memory dysfunction and more subtle distortions in memory, such as false memories, are implicated in the pathogenesis of depression and the amelioration of memory distortions may improve mood and other depressive symptoms (see Introduction). These aspects are therefore actively addressed in D-MCT and patients are given advice how to improve their memories.

In line with prior studies on the visual variant of the DRM paradigm [55-58], false memories were induced for more than half of the stimuli at baseline. Over time, both tested interventions led to a decrease in false memories, which unlikely represents a practice effect as parallel versions were used. Regarding "critical lures", the percentage of "old" ratings decreased more strongly (trend level) in the D-MCT group from pre- to post-assessment ( $61 \%$ vs. $50 \%$ ) compared to the HT group (61\% vs. $56 \%$ ) providing initial evidence that patients in the D-MCT group are less prone to false memories after treatment.

We also specifically measured high-confident judgments, as judgments that are not held in check by doubt may lead to momentous false decisions that are particularly "toxic" [61,62]. For high-confident judgments, the amount of false memories was significantly reduced over the course of treatment in the D-MCT group compared to the HT group, suggesting that a central goal of D-MCT has been met, that is, to attenuate susceptibility to false memories in depression. For both intervention groups (D-MCT and $\mathrm{HT}$ ), no significant change in confident ratings occurred for "old" items and unrelated "new" items. 
Interestingly, both groups showed an increased tendency towards "new-confident" ratings for "critical lures" at postassessment and again this effect was more pronounced in the D-MCT group compared to the HT group. This finding may indicate that D-MCT does not lead to an overall increase of doubt, but rather a better calibration of confidence. Further studies are necessary to replicate this important finding.

While false memories have often been studied in Postraumatic Stress Disorder [63-65], less attention has been given to false memories in other psychiatric diseases, including depression. Importantly, the consequences of false memories on the course of depression and the changeability of the susceptibility to false memories, for example through therapy, have not been studied so far. In our study, we confirm the high rate of false memories in depression and, for the first time, describe the amelioration of false memories using a metacognitive training approach that was designed to evoke doubt.

To date, we may only speculate on the consequences of reducing false memories in depression, but it may be postulated that the amelioration of an important cognitive distortion may contribute to overall mood stabilization in patients with depression. This would be in line with one of the purported mechanisms of change in cognitive therapy for depression, such that the reduction of "Beckian" cognitive distortions leads to symptom improvement $[66,67]$. Interestingly, decline in false memories was uncorrelated with symptom change tentatively suggesting that D-MCT improves cognitive biases and mood independently.

The current study has some limitations that we would like to acknowledge. First, we only looked at short-term changes and thus cannot determine how stable the reported changes regarding false memories were. The lack of association between improved false memories and mood awaits to be tested more rigorously. Cognitive biases are assumed to foster psychopathology and a "sleeper effect" has been supported by previous work such that this association was found only using longer (e.g., 3 years) follow-up periods [68]. Second, due to randomization we could not explore whether the effect was moderated by valence, that is, if the reduction is stronger for salient and negative-valenced material. Future studies should explore mood-congruent (negative) memories. Verbal false memory lists using lure words with validated emotional content are perhaps best suited to explore this. Third, although we assume that the effect observed was particularly due to the MCT module on memory, all D-MCT modules address thinking and cognitive biases and, therefore, the mechanism of action remains unclear. An overarching aim of D-MCT is to enhance reflection, and thus lower overconfidence in distorted selfappraisal (e.g. "I am ugly"), as well cognitive judgments (e.g., "I had a bad childhood, no one loved me). As such, this could have fostered the reduction of confidence in false memories. It would also be of interest to examine, for example, whether reduced confidence for false memories transfers to autobiographical information. We have already started to look at the modulespecific effects of MCT for psychosis by collecting cognitive and psychopathological data from participants before and after each session [69].

To conclude, the current study revealed a positive "add-on" effect of D-MCT for patients who also received an intense standard treatment in a psychosomatic hospital. Apart from the previously reported positive effects of D-MCT on depressive symptoms and psychological well-being [44], D-MCT seems to have a beneficial effect on memory biases, suggesting that D-MCT is a promising approach to correcting cognitive biases typical for depressed patients, including over-confident false judgments. Future studies should investigate whether D-MCT ameliorates other cognitive biases (e.g., OGM, MCM) as well and explore their putatively moderating role on psychopathology.

\section{Public significance statement}

Many patients with depression display false memories. Metacognitive training for depression (D-MCT) may help to reduce the susceptibility for false memories in depression.

\section{Acknowledgements}

We would like to thank Dr. Ruth Veckenstedt, Francesca Bohn, Natalia Steiner, Martina Fieker, Irmgard Musyal, Mirja Schwarz, Mario Broccucci, and Dr. Gabriela Kuhn for their help with recruitment of participants. The study was funded by a grant from the "Verein zur Förderung der Rehabilitationsforschung in Hamburg, Mecklenburg-Vorpommern und Schleswig-Holstein (vffr) [Society to promote research on rehabilitation in Hamburg, Mecklenburg-West Pomerania, and Schleswig-Holstein]" (www. reha-vffr.de); grant \#161. The vffr had no role in the design and conduct of the study; collection, management, analysis, or interpretation of the data; preparation, review, or approval of the manuscript, or the decision to submit the manuscript for publication.

\section{References}

[1] Hamdi N.R., Iacono WG. Lifetime prevalence and co-morbidity of externalizing disorders and depression in prospective assessment. Psychol Med 2014;44 (2):315-24, doi:http://dx.doi.org/10.1017/S0033291713000627.

[2] Vos T, Abajobir AA, Abate KH, Abbafati C, Abbas KM, Abd-Allah F, et al. Global, regional, and national incidence, prevalence, and years lived with disability for 328 diseases and injuries for 195 countries, 1990-2016: a systematic analysis for the Global Burden of Disease Study 2016. Lancet 2017;390(10100):1211-59, doi:http://dx.doi.org/10.1016/S0140-6736(17)32154-2.

[3] Kessler RC, Pethukova M, Sampson NA, Zaslavsky AM, Wittchen H. Twelve month and lifetime prevalence and lifetime morbid risk of anxiety and mood disorders in the United States. Int J Methods Psychiatr Res 2012;21(3):169-84, doi:http://dx.doi.org/10.1002/mpr.1359.

[4] Kessler RC. The costs of depression. Psychiatr Clin N Am 2012;35(1):1-14, doi http://dx.doi.org/10.1016/j.psc.2011.11.005

[5] McIntyre RS, Cha DS, Soczynska JK, Woldeyohannes HO, Gallaugher LA Kudlow P, et al. Cognitive deficits and functional outcomes in major depressive disorder: determinants, substrates, and treatment interventions. Depress Anxiety 2013;30(6):515-27, doi:http://dx.doi.org/10.1002/da.22063.

[6] Rock PL, Roiser JP, Riedel WJ, Blackwell AD. Cognitive impairment in depression: a systematic review and meta-analysis. Psychol Med 2014;44 (10):2029-40, doi:http://dx.doi.org/10.1017/S0033291713002535.

[7] Burt DB, Zembar MJ, Niederehe G. Depression and memory impairment: meta-analysis of the association, its pattern, and specificity. Psychol Bull 1995;117(2):285-305, doi:http://dx.doi.org/10.1037/0033-2909.117.2.285.

[8] Zhou F-C, Wang Y-Y, Zheng W, Zhang Q, Ungvari GS, Ng CH, et al. Prospective memory deficits in patients with depression: a meta-analysis. J Affect Disord 2017;220:79-85, doi:http://dx.doi.org/10.1016/j.jad.2017.05.042.

[9] Lee RSC, Hermens DF, Porter MA, Redoblado-Hodge MA. A meta-analysis of cognitive deficits in first-episode major depressive disorder. J Affect Disord 2012;140:113-24, doi:http://dx.doi.org/10.1016/j.jad.2011.10.023.

[10] Van Vreeswijk MF, De Wilde EJ. Autobiographical memory specificity, psychopathology, depressed mood and the use of the Autobiographical Memory Test: a meta-analysis. Behav Res Ther 2004;42(6):731-4, doi:http:// dx.doi.org/10.1016/S0005-7967(03)00194-3.

[11] Brittlebank AD, Scott J, Williams JM, Ferrier IN. Autobiographical memory in depression: state or trait marker? Br J Psychiatry 1993;162(1):118-21, doi: http://dx.doi.org/10.1192/bjp.162.1.118

[12] Daele T, Van Griffith, Bergh JW, Van den O, Hermans D. Overgeneral autobiographical memory predicts changes in depression in a community sample. Cognit Emotion 2014;28(7):1303-12, doi:http://dx.doi.org/10.1080/ 02699931.2013.879052.

[13] Hermans D, Vandromme H, Debeer E, Raes F, Demyttenaere K, Brunfaut E, et al. Overgeneral autobiographical memory predicts diagnostic status in depression. Behav Res Ther 2008;46(5):668-77, doi:http://dx.doi.org/10.1016/ j.brat.2008.01.018

[14] Liu Y, Zhang F, Wang Z, Cao L, Wang J, Na A, et al. Overgeneral autobiographical memory at baseline predicts depressive symptoms at follow-up in patients with first-episode depression. Psychiatry Res 2016;243:123-7, doi:http://dx doi.org/10.1016/j.psychres.2016.06.029.

[15] Sumner JA, Griffith JW, Mineka S, Rekart KN, Zinbarg RE, Craske MC Overgeneral autobiographical memory and chronic interpersonal stress as predictors of the course of depression in adolescents. Cognit Emotion 2011;25 (1):183-92, doi:http://dx.doi.org/10.1080/02699931003741566.

[16] Blaney PH. Affect and memory. A review. Psychol Bull 1986;99(2):229-46, doi: http://dx.doi.org/10.1037/0033-2909.99.2.229. 
[17] Bower GH. Mood and memory. Am Psychol 1981;36(2):129-48, doi:http://dx. doi.org/10.1037/0003-066X.36.2.129.

[18] Gaddy MA, Ingram RE. A meta-analytic review of mood-congruent implicit memory in depressed mood. Clin Psychol Rev 2014;34(5):402-16, doi:http:// dx.doi.org/10.1016/j.cpr.2014.06.001.

[19] Matt GE, Vázquez C, Campbell WK. Mood-congruent recall of affectively toned stimuli: a meta-analytic review. Clin Psychol Rev 1992;12(2):227-55, doi: http://dx.doi.org/10.1016/0272-7358(92)90116-P.

[20] Moritz S, Gläscher J, Brassen S. Investigation of mood-congruent false and true memory recognition in depression. Depress Anxiety 2005;21(1):9-17, doi: http://dx.doi.org/10.1002/da.20054.

[21] Moritz S, Voigt K, Arzola GM, Otte C. When the half-full glass is appraised as half empty and memorised as completely empty: mood-congruent true and false recognition in depression is modulated by salience. Memory 2008;16 (8):810-20, doi:http://dx.doi.org/10.1080/09658210802282708.

[22] Roediger HL, McDermott KB. Creating false memories: remembering words not presented in lists. J Exp Psychol: Learn Mem Cognit 1995;21(4):803-14, doi:http://dx.doi.org/10.1037/0278-7393.21.4.803.

[23] Howe ML, Malone C. Mood-congruent true and false memory: effects of depression. Memory 2011;19(2):192-201, doi:http://dx.doi.org/10.1080/ 09658211.2010 .544073$.

[24] Sejunaite K, Lanza C, Riepe MW. Everyday false memories in older persons with depressive disorder. Psychiatry Res 2017;261:456-63.

[25] Wittekind CE, Terfehr K, Otte C, Jelinek L, Hinkelmann K, Moritz S. Moodcongruent memory in depression - the influence of personal relevance and emotional context. Psychiatry Res 2014;215(3):606-13, doi:http://dx.doi.org/ 10.1016/j.psychres.2013.11.027.

[26] Wittekind CE, Terfehr K, Otte C, Jelinek L, Hinkelmann K, Moritz S. Moodcongruent memory in depression - the influence of personal relevance and emotional context. Psychiatry Res 2014;215(3):606-13, doi:http://dx.doi.org/ 10.1016/j.psychres.2013.11.027.

[27] Joormann J, Teachman BA, Gotlib IH. Sadder and less accurate? False memory for negative material in depression. J Abnorm Psychol 2009;118(2):412-7, doi: http://dx.doi.org/10.1037/a0015621.

[28] Ruci L, Tomes J, Zelenski J. Mood-congruent false memories in the DRM paradigm. Cognit Emotion 2009;23(6):1153-65, doi:http://dx.doi.org/ $10.1080 / 02699930802355420$.

[29] Bookbinder SH, Brainerd CJ. Emotion and false memory: the context-content paradox. Psychol Bull 2016;142(12):1315-51, doi:http://dx.doi.org/10.1037/ bul0000077.

[30] Evans VC, Iverson GL, Yatham LN, Lam RW. The relationship between neurocognitive and psychosocial functioning in major depressive disorder: a systematic review. J Clin Psychiatry 2014;75(12):1359-70, doi:http://dx.doi. org/10.4088/JCP.13r08939.

[31] Jaeger J, Berns S, Uzelac S, Davis-Conway S. Neurocognitive deficits and disability in major depressive disorder. Psychiatry Res 2006;145(1):39-48, doi:http://dx.doi.org/10.1016/j.psychres.2005.11.011.

[32] Lam RW, Kennedy SH, McIntyre RS, Khullar A. Cognitive dysfunction in major depressive disorder: effects on psychosocial functioning and implications for treatment. Can J Psychiatry 2014;59(12):649-54.

[33] Moritz S, Ferahli S, Naber D. Memory and attention performance in psychiatric patients: lack of correspondence between clinician-rated and patient-rated functioning with neuropsychological test results. J Int Neuropsychol Soc 2004;10(4):623-33, doi:http://dx.doi.org/10.1017/S1355617704104153.

[34] Kinzer A, Suhr JA. Dementia worry and its relationship to dementia exposure, psychological factors, and subjective memory concerns. Appl Neuropsychol: Adult 2016;23(3):196-204, doi:http://dx.doi.org/10.1080/ 23279095.2015.1030669.

[35] Papakostas GI. Cognitive symptoms in patients with major depressive disorder and their implications for clinical practice. J Clin Psychiatry 2014;75(1):8-14, doi:http://dx.doi.org/10.4088/JCP.13r08710.

[36] Trivedi MH, Greer TL. Cognitive dysfunction in unipolar depression: implications for treatment. J Affect Disord 2014;152-154:19-27, doi:http:// dx.doi.org/10.1016/j.jad.2013.09.012.

[37] Cuijpers P, van Straten A, Schuurmans J, van Oppen P, Hollon SD, Andersson G. Psychotherapy for chronic major depression and dysthymia: a meta-analysis. Clin Psychol Rev 2010;30(1):51-62, doi:http://dx.doi.org/10.1016/j. cpr.2009.09.003.

[38] Steinert C, Hofmann M, Kruse J, Leichsenring F. Relapse rates after psychotherapy for depression - stable long-term effects? A meta-analysis. J Affect Disord 2014;168:107-18, doi:http://dx.doi.org/10.1016/j. jad.2014.06.043.

[39] Jelinek L, Hauschildt M, Moritz S. Metakognitives Training bei Depression (DMKT). Weinheim, Deutschland: Beltz; 2015.

[40] Andreou C, Jelinek L, Balzan RP, Moritz S. Letter to the Editor: metacognitive training and metacognitive therapy. A reply to Lora Capobianco and Adrian Wells. J Behav Ther Exp Psychiatry 2018;59:162-3.

[41] Moritz S, Stöckert K, Hauschildt M, Lill H, Jelinek L, Beblo T, et al. Are we exaggerating neuropsychological impairment in depression? Reopening a closed chapter. Expert Rev Neurother 2017;17(8):839-46, doi:http://dx.doi. org/10.1080/14737175.2017.1347040.

[42] Scheurich A, Fellgiebel A, Schermuly I, Bauer S, Wölfges R, Müller MJ. Experimental evidence for a motivational origin of cognitive impairment in major depression. Psychol Med 2008;38(2):237-46, doi:http://dx.doi.org/ $\underline{10.1017 / \text { S0033291707002206. }}$
[43] Jelinek L, Otte C, Arlt S, Hauschildt M. Denkverzerrungen erkennen und korrigieren: Eine Machbarkeitsstudie zum Metakognitiven Training bei Depression (D-MKT) Zeitschrift für Psychiatrie. Psychol Psychother 2013;61 (4):247-54, doi:http://dx.doi.org/10.1024/1661-4747/a000167.

[44] Jelinek L, Hauschildt M, Wittekind CE, Schneider BC, Kriston L, Moritz S. Efficacy of metacognitive training for depression: a randomized controlled trial. Psychother Psychosom 2016;85(4):231-334, doi:http://dx.doi.org/ $10.1159 / 000443699$.

[45] Jelinek, Moritz, Hauschildt. Patients' perspectives on treatment with Metacognitive Training for Depression (D-MCT): Results on acceptability. J Affect Disord 2017:221:17-24, doi:http://dx.doi.org/10.1016/j.jad.2017.06.003.

[46] Sheehan DV, Lecrubier Y, Sheehan KH, Amorim P, Janavs J, Weiller E, et al. The Mini-International Neuropsychiatric Interview (M.I.N.I.): the development and validation of a structured diagnostic psychiatric interview for DSM-IV and ICD-10. J Clin Psychiatry 1998;59(Suppl. 20):22-33, doi:http://dx.doi.org/ 10.1016/S0924-9338(99)80239-9.

[47] Hamilton M. A rating scale for depression. J Neurol Neurosurg Psychiatry 1960;23:56-62, doi:http://dx.doi.org/10.1136/jnnp.23.1.56.

[48] Bagby RM, Ryder AG, Schuller DR, Marshall MB. The Hamilton Depression Rating Scale: has the gold standard become a lead weight? Am J Psychiatry 2004;161(12):2163-77, doi:http://dx.doi.org/10.1176/appi.ajp.161.12.2163.

[49] Worboys M. The Hamilton Rating Scale for Depression: the making of a gold standard and the unmaking of a chronic illness, 1960-1980. Chronic Illn 2013;9 (3):202-19, doi:http://dx.doi.org/10.1177/1742395312467658.

[50] Beck AT, Steer RA. Beck depression inventory. Manual. San Antonio, TX: Psychological Corporation; 1987.

[51] Hautzinger M, Bailer M, Worall H, Keller F. Beck-Depressions-Inventar (BDI). Testhandbuch [Beck Depression Inventory (BDI). Manual]. 2nd ed. Bern: Hans Huber; 1995

[52] Deese J. On the prediction of occurrence of particular verbal intrusions in immediate recall. J Exp Psychol 1959;58(1):17-22, doi:http://dx.doi.org/ 10.1037/h0046671.

[53] Miller MB, Gazzaniga MS. Creating false memories for visual scenes. Neuropsychologia 1998;36(6):513-20, doi:http://dx.doi.org/10.1016/S00283932(97)00148-6.

[54] Röder B, Rösler F. Memory for environmental sounds in sighted, congenitally blind and late blind adults: evidence for cross-modal compensation. Int J Psychophysiol 2003;50:27-39, doi:http://dx.doi.org/10.1016/S0167-8760(03) 00122-3.

[55] Jelinek L, Hottenrott B, Randjbar S, Peters MJ, Moritz S. Visual false memories in post-traumatic stress disorder (PTSD). J Behav Ther Exp Psychiatry 2009;40 (2):374-83, doi:http://dx.doi.org/10.1016/j.jbtep.2009.02.003.

[56] Moritz S, Woodward TS, Rodriguez-Raecke R. Patients with schizophrenia do not produce more false memories than controls but are more confident in them. Psychol Med 2006;36(5):659-67, doi:http://dx.doi.org/10.1017/ S0033291706007252.

[57] Otgaar H, Howe ML, Peters M, Smeets T, Moritz S. The production of spontaneous false memories across childhood. J Exp Child Psychol 2014;121 (1):28-41, doi:http://dx.doi.org/10.1016/j.jecp.2013.11.019.

[58] Schilling L, Wingenfeld K, Spitzer C, Nagel M, Moritz S. False memories and memory confidence in borderline patients. J Behav Ther Exp Psychiatry 2013;44(4):376-80, doi:http://dx.doi.org/10.1016/j.jbtep.2013.03.007.

[59] Lehrl S. Mehrfachwahl-Wortschatz-Intelligenztest: MWT-B [Multiple choice vocabulary test: MWT-B]. 3rd ed. Balingen: PERIMED-spitta; 1995.

[60] Cohen J. Statistical Power Analysis for the Behavioral Sciences. 2 rev ed. Hillsdale, N.J: Taylor \& Francis Inc; 1988.

[61] Moritz S, Van Quaquebeke N. Are you sure? Delusion conviction moderates the behavioural and emotional consequences of paranoid ideas. Cognit Neuropsychiatry 2014;19(2):164-80, doi:http://dx.doi.org/10.1080/ 13546805.2013.819781.

[62] Moritz S, Woodward TS. Metacognitive control over false memories: a key determinant of delusional thinking. Curr Psychiatry Rep 2006;8(3):184-90, doi:http://dx.doi.org/10.1007/s11920-006-0022-2.

[63] Bremner JD, Snobe KK, Kihlstrom JF. False memories in women with selfreported childhood sexual abuse: an empirical study. Psychol Sci 2000;11 (4):333-7, doi:http://dx.doi.org/10.1111/1467-9280.00266.

[64] Dasse MN, Juback SK, Mo rissette SB, Dolan SL, Weaver CA. False memory susceptibility in OEF/OIF veterans with and without PTSD. Mil Psychol 2015;27 (6):354-65, doi:http://dx.doi.org/10.1037/mil0000094.

[65] Hauschildt M, Peters MJV, Jelinek L, Moritz S. Veridical and false memory for scenic material in posttraumatic stress disorder. Conscious Cognit 2012;21 (1):80-9, doi:http://dx.doi.org/10.1016/j.concog.2011.10.013.

[66] Beck AT, Rush AJ, Shaw BF, Emery G. Cognitive therapy of depression. New York: The Guilford Press; 1979.

[67] Cristea IA, Huibers MJH, David D, Hollon SD, Andersson G, Cuijpers P. The effects of cognitive behavior therapy for adult depression on dysfunctional thinking: a meta-analysis. Clin Psychol Rev 2015;42:62-71, doi:http://dx.doi. org/10.1016/j.cpr.2015.08.003.

[68] Moritz S, Veckenstedt R, Andreou C, Bohn F, Hottenrott B, Leighton L, et al. Sustained and sleeper effects of group metacognitive training for schizophrenia a randomized clinical trial. JAMA Psychiatry 2014;71(10):110311, doi:http://dx.doi.org/10.1001/jamapsychiatry.2014.1038.

[69] Schneider BC, Cludius B, Lutz W, Moritz S, Rubel J. An investigation of modulespecific effects of metacognitive training for psychosis. Zeitschrift für Psychologie 2018. 